\title{
Myxoid chondrosarcoma of the scrotum: a case report and review of the literature
}

\author{
Carlos Enrique Mendez-Probst, MD; ${ }^{*}$ Petar Erdeljan, MD;* Mathieu Castonguay, MD; ${ }^{\dagger}$ Manal Gabril, MD; ${ }^{+}$ \\ Bret Wehrli, MD; ${ }^{\dagger}$ Hassan Razvi, MD, FRCSC
}

\begin{abstract}
Extraskeletal myxoid chondrosarcomas are relatively rare soft tissue malignant tumours, most commonly located in the extremities. Their occurrence in the genitourinary tract is extremely rare. We present a clinical case report of an extraskeletal myxoid chondrosarcoma originating in the scrotum.
\end{abstract}

Can Urol Assoc J 2010;4(4):E109-111

\section{Introduction}

Extraskeletal myxoid chondrosarcoma (EMC) is an uncommon low-grade sarcoma of the soft tissue, usually presenting as a slow-growing mass in men in their fifth to sixth decades. ${ }^{1}$ It is characterized by a multinodular growth of primitive chondroblast-like cells in an abundant myxoid matrix. It is classified as a tumour of uncertain differentiation by the World Health Organization, despite its name, since evidence of cartilaginous differentiation is lacking. Long-term diseasespecific survival is typical for this slow-growing malignant tumour; however, late local recurrence and metastases are well-described. This tumour most commonly occurs in the proximal extremities and limb girdles. ${ }^{1}$ Its appearance in the genitourinary tract is extremely rare. We present a case report and a review of the English literature.

\section{Case report}

A 75-year-old male with a medical history of an abdominal aortic aneurysm, and undergoing dialysis for chronic renal failure presented with a non tender left scrotal mass. The mass was first noted 1 year prior to consultation, with sudden rapid growth in the previous month. The patient denied any constitutional symptoms or skeletal complaints.

On physical examination, a left paratesticular solid mass, with considerable induration and fixation to the scrotal skin, was noted. No regional lymphadenopathy was noted. No other relevant physical findings were found. Ultrasound imaging showed a solid $8 \times 4.3 \times 10 \mathrm{~cm}$ mass in the paratesticular space, possibly arising from the left epididymis; both testicles were normal (Fig. 1a) (Fig. 1b).

The patient underwent scrotal exploration through an inguinal incision. The mass, although very adherent to the surrounding structures (base of the scrotum and cord structures), was clearly extratesticular. Since further dissection risked the possibility of a positive surgical margin and testicular devascularization, the testicle, chord and mass were removed en bloc.

Staging investigations including computed tomography (CT) scans of the chest, abdomen and pelvis did not reveal evidence of metastases. At 12 months post-surgery, the clinical examination and follow-up CT scan of the patient show no evidence of recurrent or metastatic disease.

\section{Pathology findings}

Gross pathological examination revealed a well-circumscribed, solid and cystic, nodular paratesticular mass, with a myxoid tan to red-brown cut surface, with hemorrhagic contents and no calcification. The testicle, epididymis and spermatic cord did not show tumour infiltration; surgical margins were negative (Fig. 2).

\section{Microscopic description}

Tumour histology on microscopy showed a lobular growth pattern which was composed of a meshwork of interlacing fascicles, single files and cords of minimally pleomorphic spindled and stellate cells (Fig. 3). Focal pseudoglandular formation was evident, set in a myxoid and sclerotic stroma. In other areas, tumour cells showed perivascular condensation forming pseudorosettes. There was moderate cytological atypia, with enlarged nuclei containing coarse chromatin and prominent nucleoli and eosinophilic cytoplasm. Mitotic figures were rare. Large intra-tumoural blood vessels contained organizing thrombi, with areas of hemorrhage and hemosiderin deposition, were noted within the tumour. There was no chondroid differentiation noted. With 


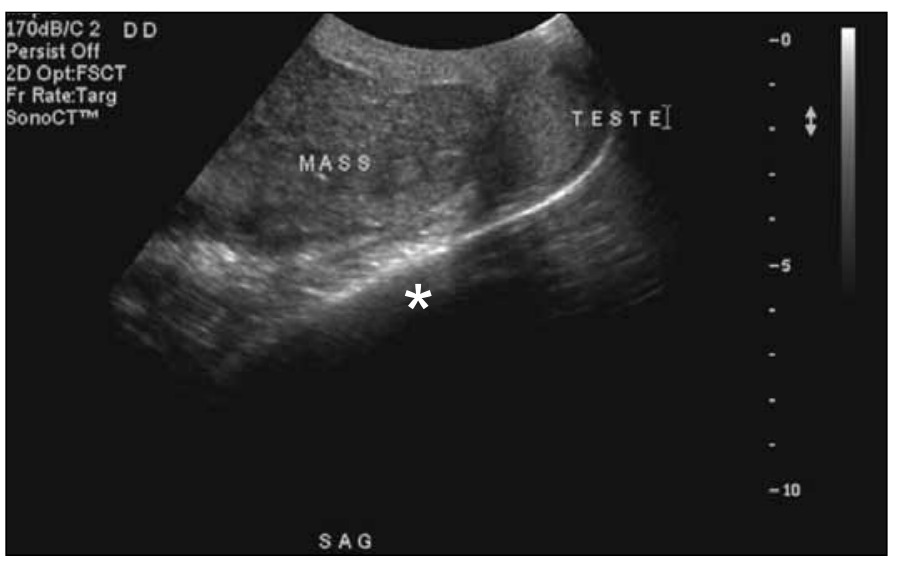

Fig. 1a. Preoperative ultrasound imaging, showing the sagittal view of the left testicle and a heterogeneous paratesticular mass. Note that in cross section, the scrotal wall bellow the mass is abnormally thickened (star).

the use of immunohistochemistry, the neoplastic cells were shown to express vimentin (Fig. 4), CD99, the neuroendocrine markers PGP9.5 and CD56, and muscle-specific actin. Expression of S-100 proteins, cytokeratin, melanoma markers (melan-A and HMB-45), CD34, ALK-1, synaptophysin, chromogranin, smooth muscle actin, myogenin, myo-D1 and desmin was not observed. Histochemically, the myxoid stroma was stained with Hale's colloidal iron which detects polyanionic compounds, such as mucopolysaccharides. Based on the above-noted features, a diagnosis of extraskeletal myxoid chondrosacoma was made.

\section{Discussion}

Extraskeletal myxoid chondrosarcoma is a rare tumour, accounting for about $2.5 \%$ of all soft tissue sarcomas. It

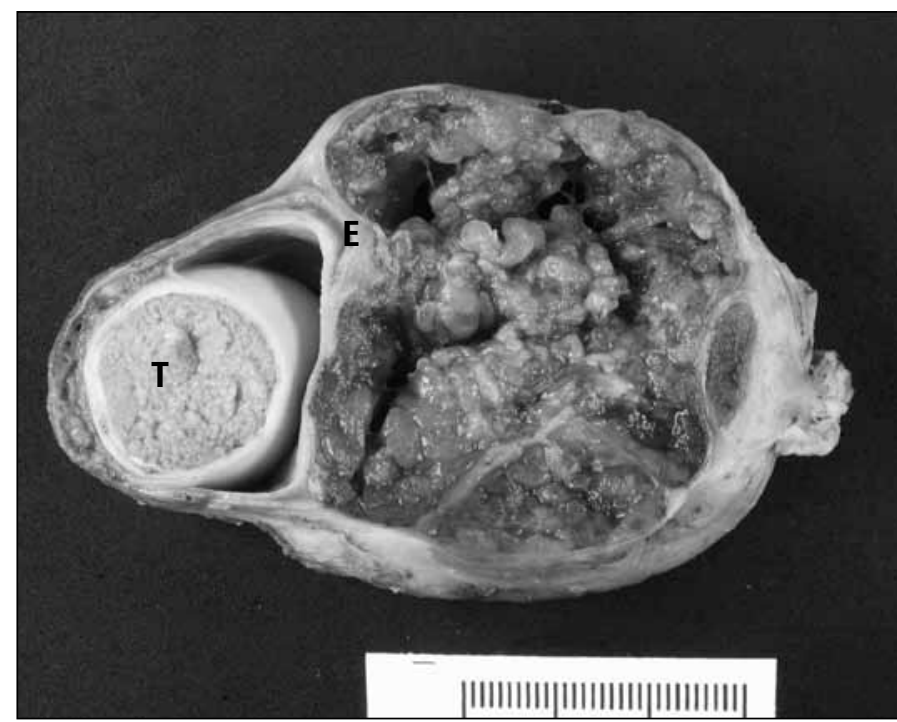

Fig. 2. Gross examination of a well-circumscribed, $9.0 \mathrm{~cm}$, solid and cystic, nodular paratesticular mass, with a myxoid tan to red-brown cut surface. The testicle $(T)$, epididymis (E) and spermatic cord (not shown), were uninvolved.

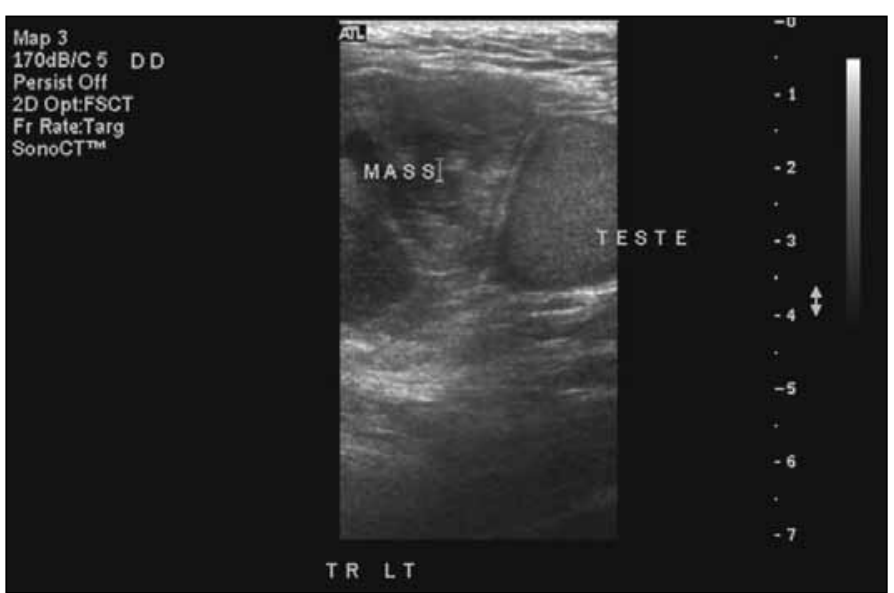

Fig. 1b. Preoperative ultrasound imaging, showing the transverse view.

commonly originates in the deep tissues of the proximal extremities and limb girdles. Genitourinary EMCs are even rarer tumours, with less than 10 cases reported since the initial report by Angervall and colleagues of a tunica vaginalis EMC in $1973 .^{2}$ Only 4 cases, including ours, involving the scrotum have been reported in the literature..$^{2-4}$ Sporadic cases involving single primary tumours in the perineum, dome of the bladder, and urethra, or as metastases to the kidney and testicle have also been reported ${ }^{5-9}$ and comprise the entire spectrum of recorded genitourinary lesions.

Most frequently, EMC arises from striated muscle, tendinous structures or deep subcutis. ${ }^{1}$ This latter location seems to be the origin in our case; however, due to the size of the mass, the exact origin of the mass could not be ascertained. It is possible that the lesion originated from the layers of the tunica vaginalis; however, in the absence of invasion of any cord structures at microscopy, this seems less likely.

Microscopically the individual tumour cells have small hyperchromatic nuclei with a small rim of eosinophilic cytoplasm which show the features of chondroblasts. Unlike chondrosarcoma of bone, there are no differentiated cartilage cells with distinct lacunae seen. ${ }^{10}$ In our case S-100 immunomarker was negative, which excludes chondroid differentiation. Morphologically, the differential diagnoses of this tumour includes chondromyxoid fibroma and myxoid liposarcoma. Other aggressive myxoid tumours, including myxoid angiomyxoma and myxoid malignant fibrous histocytoma, should be considered.

The clinical spectrum of EMC is mostly that of a slowgrowing mass, as in our case. The average size is $7 \mathrm{~cm}$, but tumours of up to $25 \mathrm{~cm}$ have been reported, ${ }^{1}$ suggesting that the patient is aware of the mass for some time before its diagnosis. Despite the delayed diagnosis, an $91 \%$ overall survival at a median of 9 years has been observed. ${ }^{1}$

The largest cohort reported on 99 EMC cases. This report highlighted that older age at diagnosis, large tumour size and tumour located in the upper extremities are adverse 


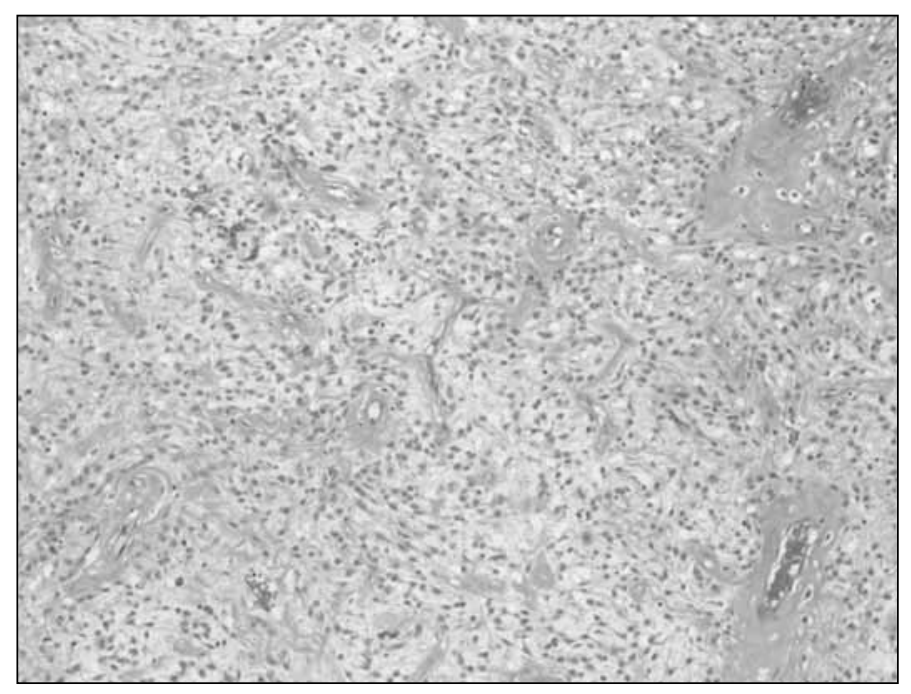

Fig. 3. Low magnification $(\times 10)$ of the tumour comprised of cords and strands of small eosinophilic cells immersed in a myxoid matrix.

prognostic factors. ${ }^{1}$ It is important to note that the above figures represent the general EMC population; in contrast, due to the paucity of scrotal EMC, we can only speculate if its clinical picture will be different. One case report described a local recurrence 4 years after a non-radical resection and no metastases. ${ }^{3}$ In another case report, a long course of recurrent disease that finally led to pulmonary metastasis more than 10 years later was documented. ${ }^{4}$

There are no reports of cancer-specific mortality with scrotal EMC; however, local recurrence seems to be common.

The cornerstone of EMC treatment is complete and often radical surgical resection of the mass, often leading to amputation when it occurs in the extremities, and radical orchiectomy in the scrotal/subinguinal region. However, this treatment has not been the subject of clinical controlled trial given the condition's rarity and thus is an extrapolation from more common sarcomas. It is to be noted that this treatment option can lead to long-term cancer-specific survival, but it appears to have no effect on local recurrence or metastatic potential, even in lieu of tumour-free microscopic margins.

The use of chemotherapy or radiotherapy for genitourinary EMC has not been reported in the literature.

\section{Conclusion}

Extraskeletal myxoid chondrosarcoma represents an extraordinary tumour in the scrotum, with only few cases currently reported in the English medical literature. Its behaviour appears to be that of a slow-growing malignancy, with the potential for local recurrence and delayed metastases.

*Division of Urology, Department of Surgery, Schulich School of Medicine \& Dentistry, The University of Western Ontario, London, ON; 'Department of Pathology, Schulich School of Medicine \& Dentistry, The University of Western Ontario, London, ON

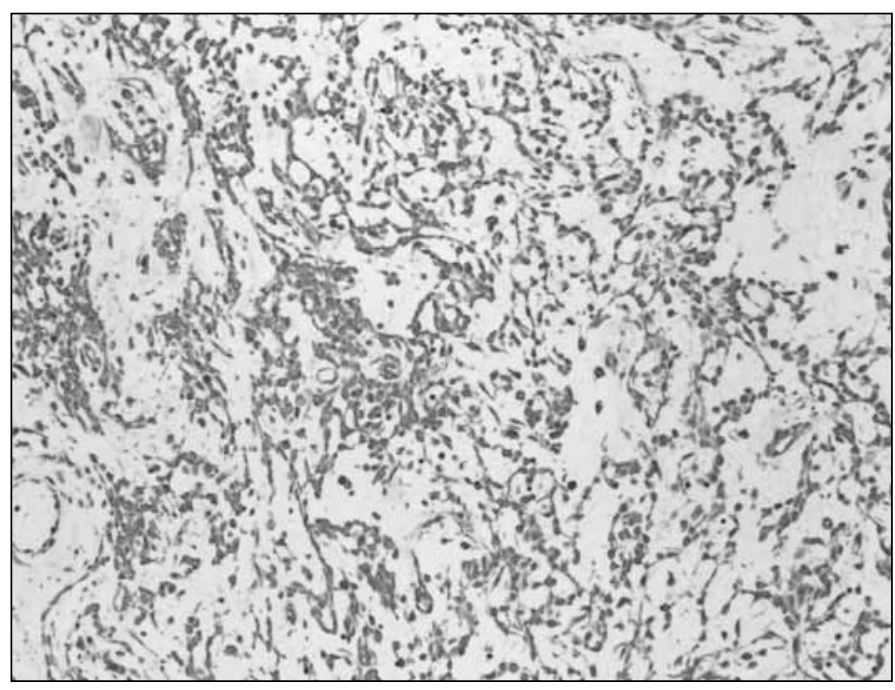

Fig. 4. Low magnification $(\times 10)$ of positive immunoreactivity for vimentin.

Competing interests: None declared.

This paper has been peer-reviewed.

\section{References}

1. Meis-Kindblom JM, Bergh P, Gunterberg B et al. Extraskeletal myxoid chondrosarcoma: a reappraisal of its morphologic spectrum and prognostic factors based on 117 cases. Am J Surg Pathol 1999;23:636-50.

2. Angervall L, Enerbäck L, Knutson H. Chondrosarcoma of soft tissue origin. Cancer 1973;32:507-13.

3. MacDonald GR Jr, D'Ambrosio P, Marshall DP. Chondrosarcoma of spermatic cord. Urology 1977;9:439-41.

4. Christenson PJ, $0^{\prime}$ Connell KJ. Metastatic extraosseous myxoid chondrosarcoma of spermatic cord. Urology 1985;26:301-3.

5. Morimura Y, Fujimori K, Sato T, et al. Imprint cytology of extraskeletal mesenchymal chondrosarcoma of the perineum: a case report. Acta Cytol 2004;48:649-52.

6. Goldenberg RR, Cohen P, Steinlauf P. Chondrosarcoma of the extraskeletal soft tissues. A report of seven cases and review of the literature. J Bone Joint Surg Am 1967;49:1487-507.

7. Yi JW, Park YK, Choi YM, et al. Bulbous urethra involved in perineal extraskeletal myxoid chondrosarcoma in a child. Int J Urol 2004;11:436-9.

8. Kauffman SL, Stout AP. Extraskeletal osteogenic sarcomas and chondrosarcomas in children. Cancer 1963;16:432-9.

9. Gupta R, Sharma MC, Attri S, et al. Testicular metastasis of extraskeletal myxoid chondrosarcoma: report of first case. Urology 2008;71:984.el-4.

10. Weiss SW, Goldblum JR. Extraskeletal myxoid chondrosarcoma. In: Weiss SW, Goldblum JR, eds. Enzinger and Weiss's Soft Tissue Tumours. 4th edition. Philadelphia, PA: Mosby; 2001:1368-79.

Correspondence: Dr. Hassan Razvi, Urology, St. Joseph's Hospital, 268 Grosvenor St., London, ON N6A 4V2; fax: 519-646-6037; hrazvi@uwo.ca 